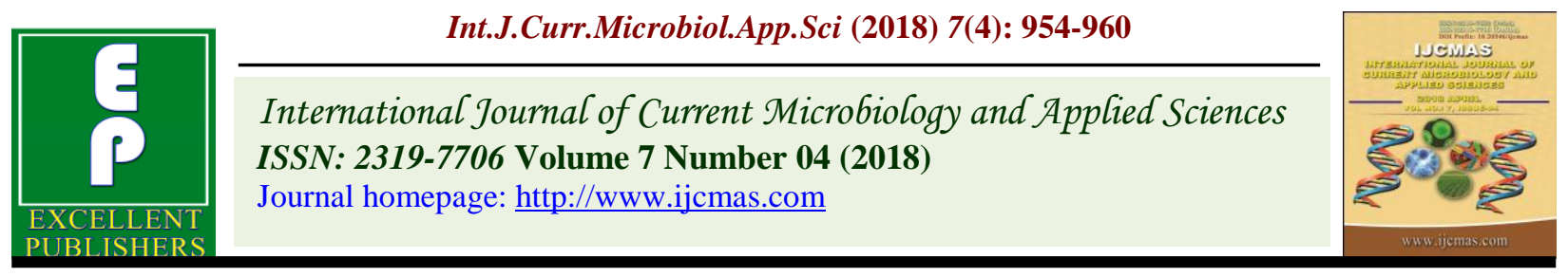

Original Research Article

https://doi.org/10.20546/ijcmas.2018.704.101

\title{
Combining Ability Analysis for Seed Yield and Its Component Traits with Diverse CMS Sources in Sunflower (Helianthus annuus L.)
}

\author{
Sukanya Biradar, A.G. Vijaykumar" and G.K. Naidu \\ Department of Genetics and Plant Breeding, College of Agriculture, (University of \\ Agricultural Sciences, Dharwad), Vijayapura-586101, Karnataka, India \\ *Corresponding author
}

\section{A B S T R A C T}

\section{Keywords}

Sunflower, Diverse CMS

source, Combining

ability, Line x tester

analysis, Helianthus

petiolaris sp. fallax, $H$.

annuus sp. lenticularis

Article Info

Accepted:

10 March 2018

Available Online:

10 April 2018
Two new CMS sources (CMS PEF and CMS I) along with the conventional PET source and 10 new restorer lines were crossed in a line $\mathrm{x}$ tester fashion to elucidate the information on the combining ability for seed yield and its components traits. A total of 40 crosses evaluated for 12 different quantitative traits. Among the lines CMS 234A and IMS 852A exhibited significant gca effects for most of the characters studied and were found to be the best combiners. Likewise, testers IB-104, DSR-35 and NS-15 were found to be good general combiners for most of the traits studied. The estimates of variance components revealed predominance of non-additive gene action for most of the characters studied.

\section{Introduction}

Sunflower (Helinathus annuus L.) is an important oilseed crop of India. To develop sunflower hybrids with improved yield potential, the choice of parents through careful and critical evaluation is of very importance in order to improve productivity and total production. The seed yield and yield attributing characters show polygenic inheritance and thus susceptible to environmental fluctuations. Therefore, selection of parents for hybridization is a complex problem. Hybrids using lines developed based on heterosis are preferred by farmers due to their high yielding performance, quality and uniformity. The concept of general combining ability and specific combining ability (Sprague and Tatum, 1942) reveals additive and nonadditive gene actions, respectively. This helps the breeders to assess the parents for adoption in heterosis breeding programme. The choice of the parents is governed by per se performance of the parents and behavior of the parents in respective hybrid combination. Some idea on the usefulness of parents may be obtained from their individual performance particularly in respect of yield components (Gilbert, 1958). The mode of gene action depends upon the genetic structure and extent of divergence between the parents involved. 
Therefore, it is necessary to estimate the genetic potentialities of parents in hybrid combinations through systematic studies with regard to general combining ability and specific combining ability. A wide range of variability and cytoplasmic male sterility are available in sunflower. With a view to identify the lines with good combining ability and to identify the good specific crosses for further exploitation, the present investigation was under taken.

\section{Materials and Methods}

The present study was carried out during late kharif 2016 at Regional Agricultural Research Station, Vijayapura (Karnataka) India, located in the northern dry zone of Karnataka at an altitude of $593 \mathrm{~m}$ from mean sea level. Crossing programme was done in summer 2015-16 and evaluation of parents and $F_{1}$ 's was taken up in late kharif 2016. All the 100 experimental hybrids produced were sown along with their parents and commercial checks viz., DSFH-3, KBSH-53, KBSH-41 in a Randomised Block Design (RBD) with two replications. The plot size for each entry consisted of two rows (0.6 meter) in each replication. Based on the fertility restoration studies only 40 hybrids that satisfied the $\mathrm{L} \times \mathrm{T}$ requirements were used for assessing heterosis and combining ability.

The parent materials for the present study consisted of four cytoplasmic male sterile lines viz., FMS 852A (CMS PEF), IMS 852A (CMS I), CMS 302A (PET 1) and CMS 234A (PET 1) and ten new restorer lines viz., RHA1-1, GP-9, GP-5, DSR-107, NS-15, DSR-35, DSR-37, IB-60, IB-03 and IB-104 were used in the investigation. The following observations of twelve different quantitative characters were recorded: viz., days to 50 percent flowering, Days to maturity, plant height $(\mathrm{cm})$, head diameter $(\mathrm{cm})$, Per cent seed filling (\%), volume weight $\mathrm{g} / \mathrm{cc})$, test weight $(\mathrm{g})$, seed yield per plant $(\mathrm{g})$, seed yield $(\mathrm{kg} / \mathrm{ha})$, oil content $(\%)$, hull percent $(\%)$ and oil yield $(\mathrm{kg} / \mathrm{ha})$. The data were analyzed by $\mathrm{L}$ $\times \mathrm{T}$.

\section{Results and Discussion}

The analysis of variance for combining ability of parents and crosses for different yield component traits in line $\times$ tester design (Table 1) indicated the significant variation for all the traits indicating the existing of genetic diversity in the parental material and varying performance of the cross combinations. The contribution of testers as compared to lines was more for days to $50 \%$ flowering, days to maturity, plant height $(\mathrm{cm})$, head diameter $(\mathrm{cm})$, per cent seed filling, volume weight $(\mathrm{g} / \mathrm{cc})$, test weight $(\mathrm{g})$, seed yield per plant $(\mathrm{g})$, seed yield $(\mathrm{kg} / \mathrm{ha})$, oil content $(\%)$ and oil yield (kg/ha). The contribution of lines as compared to testers was more for hull content $(\%)$. The variance due to line $\mathrm{x}$ tester was highly significant for all the traits indicating that the testers were highly divergent from lines. The SCA variance was higher than GCA variance for all the traits studied indicating predominance of non-additive type of gene action which is in agreement with the findings of Yogesh et al., (2007), Channamma (2009), Mohanasundaram et al., (2010), Kang et al., (2013) and Khandagale et al., (2014). The ratio of GCA/SCA variance observed to be higher for test weight (g) and oil content (\%) as compared to other traits.

The general combining ability effects (Table 2) indicated that CMS-234A was good general combiner as evident from its significant gca effect in desirable direction for plant height $(\mathrm{cm})$, volume weight $(\mathrm{g} / \mathrm{cc})$, test weight $(\mathrm{g})$, seed yield per plant, seed yield $(\mathrm{kg} / \mathrm{ha})$, hull content (\%) and oil yield (kg/ha). For earliness, the line FMS 852A and the tester RHA-1-1 were found to be good general combiners. 
Table.1 Analysis of variance for combining ability of CMS and restorer lines and CMS lines for different yield and yield components in sunflower

\begin{tabular}{|c|c|c|c|c|c|c|c|c|c|c|c|c|c|}
\hline Sources & df & $\begin{array}{c}\text { Days to } 50 \% \\
\text { flowering }\end{array}$ & $\begin{array}{l}\text { Days to } \\
\text { maturity }\end{array}$ & $\begin{array}{l}\text { Plant } \\
\text { height } \\
\text { (cm) }\end{array}$ & $\begin{array}{c}\text { Head } \\
\text { diameter } \\
\text { (cm) }\end{array}$ & $\begin{array}{l}\text { Per cent } \\
\text { seed } \\
\text { filling }\end{array}$ & $\begin{array}{c}\text { Volume } \\
\text { weight (g) }\end{array}$ & $\begin{array}{c}\text { Test weight } \\
\text { (g/100 } \\
\text { seeds) }\end{array}$ & $\begin{array}{c}\text { Seed } \\
\text { yield/plant } \\
\text { (g) }\end{array}$ & $\begin{array}{c}\text { Seed yield } \\
\text { (kg/ha) }\end{array}$ & $\begin{array}{c}\text { Hull } \\
\text { content } \\
(\%)\end{array}$ & $\begin{array}{c}\text { Oil } \\
\text { content } \\
(\%)\end{array}$ & $\begin{array}{l}\text { Oil yield } \\
\text { (kg/ha) }\end{array}$ \\
\hline Replication & 1 & 0.45 & 3.2 & 3.05 & 0.002 & 0.61 & 0.04 & 0.03 & 0.00 & 7.50 & 1.12 & 0.28 & 145.93 \\
\hline Crosses & 39 & $620.95^{* *}$ & $404.25^{* *}$ & $411.37 * *$ & $8.71 * *$ & $4.21 * *$ & $4.17 * *$ & $0.79 * *$ & $17.97 * *$ & $268566.62 * *$ & $4.31 * *$ & $5.11 * *$ & $44423.53 * *$ \\
\hline Line Effect & 3 & 0.45 & 427.68 & 191.26 & 3.99 & 4.97 & 0.53 & 0.43 & 10.81 & 161602.24 & 5.71 & 10.50 & 20771.96 \\
\hline Tester Effect & 9 & 94.45 & 441.55 & 432.51 & 7.55 & 5.20 & 7.80 & 1.58 & 31.18 & 465869.38 & 1.42 & 4.62 & 75735.89 \\
\hline $\begin{array}{l}\text { Line } x \text { Tester } \\
\text { effect }\end{array}$ & 27 & $526.05^{* * *}$ & $389.22 * *$ & $428.77 * *$ & $9.62 * *$ & $3.80 * *$ & $3.37 * *$ & $0.56^{* *}$ & $14.36^{* *}$ & $214683.96 * *$ & $5.11 * *$ & $4.67 * *$ & $36614.03 * *$ \\
\hline Error & 39 & 24.55 & 5.76 & 0.50 & 0.02 & 0.15 & 0.03 & 0.02 & 0.02 & 287.07 & 0.23 & 0.25 & 222.31 \\
\hline Total & 79 & 645.95 & 202.45 & 203.36 & 4.31 & 2.16 & 2.07 & 0.39 & 8.88 & 132725.34 & 2.25 & 2.65 & 22042.20 \\
\hline var GCA & & -1.011 & 3.24 & -8.35 & -0.27 & $0.09 *$ & $0.05^{*}$ & $0.03 * *$ & $0.47 *$ & $7075.13 *$ & -0.11 & 0.20 * & $831.42 *$ \\
\hline var SCA & & $9.41 * *$ & $192.17 * *$ & $214.16^{* *}$ & $4.79 * *$ & $1.81 * *$ & $1.67 * *$ & $0.27 * *$ & $7.17 * *$ & $107200.99 * *$ & $2.43 * *$ & $2.21 * *$ & $18199.55^{* *}$ \\
\hline $\begin{array}{l}\text { var GCA/ var } \\
\text { SCA }\end{array}$ & & -0.11 & 0.017 & -0.04 & -0.057 & 0.05 & 0.03 & 0.11 & 0.06 & 0.06 & 0.04 & 0.09 & 0.05 \\
\hline \multicolumn{14}{|c|}{ Contribution $\%$ of } \\
\hline Lines & & 0.07 & 8.14 & 3.57 & 3.53 & 9.06 & 0.99 & 4.22 & 4.62 & 4.62 & 10.19 & 15.8 & 3.59 \\
\hline Testers & & 15.21 & 25.2 & 24.26 & 19.99 & 28.5 & 43.11 & 46.25 & 40.02 & 40.03 & 7.61 & 20.86 & 39.34 \\
\hline $\begin{array}{l}\text { Lines } x \\
\text { Testers }\end{array}$ & & 84.71 & 66.65 & 72.16 & 76.47 & 62.42 & 55.89 & 49.52 & 55.34 & 55.34 & 82.19 & 63.32 & 57.06 \\
\hline
\end{tabular}


Table.2 General combining ability effects (gca) of parents for seed yield and its component traits in sunflower

\begin{tabular}{|c|c|c|c|c|c|c|c|c|c|c|c|c|c|}
\hline SI & Sources & DF & DM & PH (cm) & HD (cm) & PSF (\%) & VW (g/cc) & HSW (g) & SPP (g) & SY (y/ha) & HC (\%) & $\mathrm{OC}(\%)$ & OY (g/ha) \\
\hline & \multicolumn{13}{|l|}{ Female lines } \\
\hline 1 & FMS 852A & -0.03 & $-6.93 * *$ & $-1.37 * *$ & $0.48 * *$ & -0.14 & -0.06 & $-0.10 * *$ & $-0.68 * *$ & $-83.41 * *$ & 0.10 & $0.25 *$ & $-24.71 * *$ \\
\hline 2 & IMS 852A & -0.03 & $2.33 * *$ & $4.44 * *$ & $-0.37 * *$ & $0.54 * *$ & -0.07 & $0.14 * *$ & $-0.47 * *$ & $-57.75 * *$ & $-0.27 *$ & $0.61 * *$ & -4.39 \\
\hline 3 & CMS 302A & 0.13 & $1.97 * *$ & $-2.62 * *$ & $0.27 * *$ & $0.22 *$ & $-0.11 * *$ & $-0.15 * *$ & $0.21 * *$ & $25.96 * *$ & $0.70 * *$ & $-1.05 * *$ & $-17.56 * *$ \\
\hline \multirow[t]{5}{*}{4} & CMS 234A & -0.07 & $2.63 * *$ & $-0.44 * *$ & $-0.38 * *$ & $-0.61 * *$ & $0.24 * *$ & $0.11 * *$ & $0.94 * *$ & $115.20 * *$ & $-0.52 * *$ & 0.19 & $46.66 * *$ \\
\hline & SE for lines & 0.18 & 0.49 & 0.15 & 0.04 & 0.09 & 0.04 & 0.03 & 0.03 & 3.75 & 0.12 & 0.11 & 3.28 \\
\hline & CD@ $5 \%$ & 0.37 & 0.99 & 0.31 & 0.08 & 0.18 & 0.08 & 0.06 & 0.06 & 7.59 & 0.22 & 0.23 & 6.63 \\
\hline & CD @ $1 \%$ & 0.49 & 1.34 & 0.41 & 0.11 & 0.25 & 0.11 & 0.07 & 0.08 & 10.16 & 0.30 & 0.30 & 8.88 \\
\hline & \multicolumn{13}{|l|}{ Male lines } \\
\hline 1 & RHA-1-1 & $1.10^{* *}$ & $-20.98 * *$ & $-3.89 * *$ & $-0.86^{* *}$ & -0.10 & $-0.17 *$ & $-0.19 * *$ & $-3.53 * *$ & $-432.08 * *$ & $0.38 *$ & $-0.54 * *$ & $-173.16 * *$ \\
\hline 2 & GP-9 & $-1.90 * *$ & $2.03 *$ & $-4.58 * *$ & $0.19 * *$ & $0.85^{* *}$ & $-0.88 * *$ & $-0.29 * *$ & $-1.11 * *$ & $-135.66 * *$ & 0.05 & -0.12 & $-55.21 * *$ \\
\hline 3 & GP-5 & $-1.65 * *$ & $2.53 * *$ & $4.07 * *$ & $0.31 * *$ & $-1.21 * *$ & $-0.54 * *$ & -0.06 & $1.01 * *$ & $124.06 * *$ & -0.27 & -0.14 & $39.63 * *$ \\
\hline 4 & DSR-107 & -0.03 & $3.65 * *$ & $-8.95 * *$ & $-1.87 * *$ & $1.25 * *$ & $0.45 * *$ & $0.34 * *$ & $0.45 * *$ & $55.30 * *$ & $-0.83 * *$ & -0.17 & $16.57 * *$ \\
\hline 5 & NS-15 & $0.98 * *$ & $2.78 * *$ & $4.82 * *$ & $-0.51 * *$ & $-0.54 * *$ & $1.16 * *$ & $0.32 * *$ & $1.09 * *$ & $133.21 * *$ & 0.02 & $1.92 * *$ & $95.77 * *$ \\
\hline 6 & DSR-35 & $1.23 * *$ & $2.40 * *$ & $2.65 * *$ & $-0.39 * *$ & $0.34 *$ & $1.89 * *$ & $0.87 * *$ & $2.84 * *$ & $347.15 * *$ & 0.01 & 0.27 & $132.62 * *$ \\
\hline 7 & DSR-37 & 0.23 & $3.53 * *$ & $5.53 * *$ & $0.28 * *$ & $0.55 * *$ & $-0.98 * *$ & $-0.66 * *$ & -0.42 & $-51.65 * *$ & -0.31 & $-0.37 *$ & $-28.67 * *$ \\
\hline 8 & IB-60 & -0.53 & 1.53 & $12.9 * *$ & $0.48 * *$ & -0.23 & $-0.59 * *$ & -0.02 & $-2.02 * *$ & $-247.22 * *$ & $0.44 *$ & $-0.92 * *$ & $-105.79 * *$ \\
\hline 9 & IB-03 & -0.53 & 0.40 & $-11.1 * *$ & $1.73 * *$ & 0.22 & $-0.93 * *$ & $-0.47 * *$ & -0.76 & $-92.89 * *$ & $0.62 * *$ & 0.26 & $-26.05 * *$ \\
\hline 10 & IB-104 & $1.10 * *$ & $2.15 * *$ & $-1.46^{* *}$ & $0.64 * *$ & $-1.12 * *$ & $0.58 * *$ & $0.18 * *$ & $2.45 * *$ & $299.78 * *$ & -0.12 & -0.18 & $104.28 * *$ \\
\hline & SE for testers & 0.29 & 0.78 & 0.24 & 0.07 & 0.14 & 0.06 & 0.04 & 0.05 & 5.93 & 0.18 & 0.17 & 5.18 \\
\hline & CD@ $5 \%$ & 0.58 & 1.59 & 0.48 & 0.14 & 0.29 & 0.13 & 0.09 & 0.09 & 12.01 & 0.36 & 0.36 & 10.48 \\
\hline & CD@ $1 \%$ & 0.78 & 2.12 & 0.65 & 0.18 & 0.39 & 0.17 & 0.12 & 0.13 & 16.07 & 0.48 & 0.48 & 14.04 \\
\hline
\end{tabular}

\footnotetext{
* - Significant at $5 \% \quad * *$ - Significant at $1 \%$
} 
Table.3 Specific combining ability (sca) effects for seed yield and its component traits in sunflower

\begin{tabular}{|c|}
\hline SI No. \\
\hline 1 \\
\hline 2 \\
\hline 3 \\
\hline 4 \\
\hline 5 \\
\hline 6 \\
\hline 7 \\
\hline 8 \\
\hline 9 \\
\hline 10 \\
\hline 11 \\
\hline 12 \\
\hline 13 \\
\hline 14 \\
\hline 15 \\
\hline 16 \\
\hline 17 \\
\hline 18 \\
\hline 19 \\
\hline 20 \\
\hline 21 \\
\hline 22 \\
\hline 23 \\
\hline 24 \\
\hline 25 \\
\hline 26 \\
\hline 27 \\
\hline 28 \\
\hline 29 \\
\hline
\end{tabular}

\begin{tabular}{|c|c|c|c|c|c|c|c|c|c|c|c|c|}
\hline Sources & DF & DM & PH (cm) & HD $(\mathbf{c m})$ & PSF (\%) & VW (g/cc) & HSW (g) & SPP (g) & SY (kg/ha) & $\mathrm{HC}(\%)$ & $\mathrm{OC}(\%)$ & OY (kg/ha) \\
\hline FMS $852 \mathrm{~A} \times$ RHA-1-1 & $1.90 * *$ & $-5.33 *$ & $-4.36 * *$ & $-1.44 * *$ & 0.15 & $-0.51 * *$ & $-0.38 * *$ & $-5.41 * *$ & $-660.66 * *$ & $1.57 * *$ & -0.07 & $-239.54 * *$ \\
\hline FMS 852A x GP-9 & $-3.60 * *$ & $6.68 * *$ & $-7.38 * *$ & $2.17 * *$ & $0.63^{*}$ & $1.56 * *$ & 0.07 & $3.77 * *$ & $460.82 * *$ & $0.75^{*}$ & $0.85^{*}$ & $193.09 * *$ \\
\hline FMS 852A x GP-5 & $-3.35 * *$ & $7.18 * *$ & $10.18 * *$ & $0.44 * *$ & $1.80 * *$ & -0.03 & $-0.52 * *$ & $-0.61 * *$ & $-73.96 * *$ & 0.47 & -0.28 & $-32.79 * *$ \\
\hline FMS 852A x DSR-107 & $3.03 * *$ & $7.05 * *$ & -0.01 & 0.18 & 0.19 & $-1.78^{* *}$ & $-0.22 *$ & $-5.04 * *$ & $-616.35 * *$ & $-0.86^{*}$ & $-0.79 *$ & $-242.31 * *$ \\
\hline FMS 852A x NS-15 & 0.03 & $4.93 * *$ & $-9.13 * *$ & $-0.34 *$ & $-2.62 * *$ & $1.21 * *$ & $0.85 * *$ & $-0.43 * *$ & $-52.56 * *$ & $-2.56 * *$ & -0.26 & $-27.44^{*}$ \\
\hline FMS 852A x DSR-35 & $-1.23^{*}$ & $5.80 * *$ & $7.99 * *$ & $-3.26 * *$ & $0.62 *$ & $0.83 * *$ & $0.30 * *$ & $1.57 * *$ & $191.90 * *$ & $1.45 * *$ & -0.52 & $57.58 * *$ \\
\hline FMS 852A x DSR-37 & $2.78^{* * *}$ & $8.18 * *$ & $-3.09 * *$ & $-2.87 * *$ & $-1.47 * *$ & -0.19 & $0.33 * *$ & $0.73 * *$ & $89.50^{* *}$ & $-1.89 * *$ & -0.71 & 15.51 \\
\hline FMS 852A x IB-60 & 1.03 & $7.18 * *$ & $1.70 * *$ & $2.63 * *$ & $0.62 *$ & $0.57 * *$ & $0.49 * *$ & $3.23 * *$ & $395.13 * *$ & $-0.74 *$ & 0.40 & $145.89^{* *}$ \\
\hline FMS $852 \mathrm{~A} \times \mathrm{IB}-03$ & 1.03 & $6.30 * *$ & $20.55 * *$ & $1.58^{* *}$ & $0.87 * *$ & $-1.59 * *$ & $-0.5^{* *}$ & $0.42 * *$ & $51.35^{* *}$ & $0.89 *$ & 0.58 & $28.41 * *$ \\
\hline FMS 852A x IB-104 & $-1.60 * *$ & $6.05 * *$ & $-16.45 * *$ & $0.92 * *$ & $-0.79 *$ & -0.06 & $-0.41 * *$ & $1.76^{* *}$ & $214.82 * *$ & $0.92 *$ & $0.79^{*}$ & $101.59^{* *}$ \\
\hline IMS 852A x RHA-1-1 & $-3.60 * *$ & $19.43^{* *}$ & $2.12 * *$ & $-1.03 * *$ & -0.44 & -0.09 & $-0.23^{*}$ & -0.17 & -20.17 & -0.15 & -0.34 & -16.38 \\
\hline IMS 852A x GP-9 & $4.40^{* *}$ & -1.58 & $15.31 * *$ & $-0.33 *$ & -0.10 & $-0.82 * *$ & $0.27 * *$ & $-2.04 * *$ & $-249.34 * *$ & $-1.63 * *$ & $2.85 * *$ & $-38.08 * *$ \\
\hline IMS 852A x GP-5 & $3.15 * *$ & $-3.58 *$ & $22.96 * *$ & -0.05 & $-1.39 * *$ & $0.39 * *$ & $0.83 * *$ & $-1.52 * *$ & $-185.17 * *$ & $-3.70 * *$ & -0.08 & $-71.47 * *$ \\
\hline IMS 852A x DSR-107 & $-2.48 * *$ & -2.2 & $-2.08 * *$ & $-0.91 * *$ & $1.26 * *$ & $1.24 * *$ & 0.13 & $3.35 * *$ & $409.14 * *$ & -0.44 & $1.22 * *$ & $182.39 * *$ \\
\hline IMS 852A x NS-15 & -0.48 & -0.83 & 0.31 & $2.38 * *$ & 0.55 & $0.83 * *$ & $0.26 * *$ & $2.36^{* *}$ & $288.48^{* *}$ & $1.36 * *$ & $-1.03 * *$ & $82.31 * *$ \\
\hline IMS 852A x DSR-35 & -0.73 & -2.45 & $3.57 * *$ & $-1.85 * *$ & $-1.21 * *$ & $0.85^{* *}$ & -0.14 & 0.11 & 13.44 & 0.27 & $1.66^{* *}$ & $50.33 * *$ \\
\hline IMS 852A x DSR-37 & $-1.23 *$ & -3.00 & $8.59 * *$ & $0.79 * *$ & $1.25^{* *}$ & $0.68 * *$ & $0.27 * *$ & $1.32 * *$ & $161.64 * *$ & $1.19 * *$ & $1.09 * *$ & $83.61 * *$ \\
\hline IMS 852A x IB-60 & $-4.98 * *$ & $-3.58 *$ & $-36.37 * *$ & -0.06 & $0.83 * *$ & $-0.36^{* *}$ & 0.15 & $-2.53 * *$ & $-308.93 * *$ & $1.39 * *$ & $-3.29 * *$ & $-182.00 * *$ \\
\hline IMS 852A x IB-03 & $3.03 * *$ & -0.95 & $-18.62 * *$ & $-1.81 * *$ & $-1.87 * *$ & $-0.52 * *$ & -0.05 & $-2.29 * *$ & $-279.91 * *$ & -0.59 & $-2.14 * *$ & $-154.48 * *$ \\
\hline IMS 852A x IB-104 & $2.90 * *$ & -1.20 & $4.19 * *$ & $2.88^{* *}$ & $1.12 * *$ & $-2.19 * *$ & $-0.95 * *$ & $1.39 * *$ & $170.81 * *$ & $2.29 * *$ & 0.07 & $63.77 * *$ \\
\hline CMS 302A x RHA-1-1 & $1.25^{*}$ & $19.78 * *$ & $2.69 * *$ & $0.78^{* *}$ & $-1.47 * *$ & $-0.35 * *$ & 0.01 & $4.55^{* *}$ & $556.16^{* *}$ & $-0.98 * *$ & $-0.77^{*}$ & $183.23 * *$ \\
\hline CMS 302A x GP-9 & $-4.75 * *$ & $-4.23 *$ & $-1.43 * *$ & $-3.48 * *$ & -0.19 & $-1.04 * *$ & $-0.44 * *$ & $-0.88 * *$ & $-106.97 * *$ & 0.19 & $-2.07 * *$ & $-80.09 * *$ \\
\hline CMS 302A x GP-5 & -1.00 & -0.23 & $-26.08 * *$ & $1.75 * *$ & $0.67 *$ & $0.57 * *$ & 0.17 & $1.35 * *$ & $165.01 * *$ & $1.22 * *$ & -0.05 & $57.63 * *$ \\
\hline CMS 302A x DSR-107 & $1.88^{* *}$ & -1.35 & $-3.71 * *$ & $1.489 * *$ & $-1.70^{* *}$ & -0.17 & -0.18 & $1.56^{* * *}$ & $191.02 * *$ & $1.69 * *$ & $1.00 * *$ & $93.35 * *$ \\
\hline CMS 302A x NS-15 & $-1.63 * *$ & $-3.48 *$ & $10.08 * *$ & $-0.83 * *$ & $1.36^{* *}$ & $1.16^{* * *}$ & -0.10 & $-1.63 * *$ & $-198.64 * *$ & -0.11 & $1.74 * *$ & $-32.06 * *$ \\
\hline CMS 302A x DSR-35 & $1.63 * *$ & -2.10 & $-5.71 * *$ & $1.95 * *$ & -0.58 & $-1.16 * *$ & -0.05 & -0.12 & -15.28 & 0.49 & $-0.97 * *$ & $-34.28 * *$ \\
\hline CMS 302A x DSR-37 & $-2.38 * *$ & -1.23 & $-5.39 * *$ & 0.14 & 0.11 & $0.41 * *$ & $0.22 *$ & $-0.51 * *$ & $-62.63 * *$ & 0.01 & $0.83^{*}$ & -1.62 \\
\hline CMS 302A x IB-60 & $3.38 * *$ & -2.23 & $22.80 * *$ & $-1.16 * *$ & $1.30 * *$ & $-0.98 * *$ & $-0.66^{* *}$ & $-3.31 * *$ & $-404.91 * *$ & -0.39 & 0.34 & $-137.34 * *$ \\
\hline CMS 302A x IB-03 & $1.88 * *$ & -2.60 & $-10.75 * *$ & $-0.91 * *$ & 0.45 & $0.41 * *$ & $0.29 * *$ & $-0.73 * *$ & $-88.63 * *$ & -0.41 & -0.25 & $-38.73 * *$ \\
\hline
\end{tabular}

Contd...

\begin{tabular}{|c|c|c|c|c|c|c|c|c|c|c|c|c|c|}
\hline SI No. & Sources & DF & DM & PH (cm) & $\mathrm{HD}(\mathrm{cm})$ & PSF $(\%)$ & VW (g/cc) & HSW (g) & SPP (g) & SY (y/ha) & HC $(\%)$ & OC $(\%)$ & OY (kg/ha) \\
\hline 30 & CMS 302A x IB-104 & -0.25 & -2.35 & $17.50 * *$ & $0.28 *$ & 0.04 & $1.15 * *$ & $0.74 * *$ & $-0.29 * *$ & $-35.12 * *$ & $-1.73 * *$ & 0.19 & -10.09 \\
\hline 31 & CMS 234A x RHA-1-1 & 0.45 & $20.13^{* *}$ & -0.45 & $1.69 * *$ & $1.77^{* *}$ & $-0.94 * *$ & 0.60 ** & $1.02 * *$ & $124.67 * *$ & -0.45 & $1.178^{* *}$ & $72.69^{* *}$ \\
\hline 32 & CMS 234A x GP-9 & $3.95 * *$ & -0.88 & $-6.51^{* *}$ & $1.64 * *$ & -0.35 & $0.31 *$ & 0.10 & $-0.85^{* *}$ & $-104.50 * *$ & 0.68 & $-1.63^{* *}$ & $-74.91 * *$ \\
\hline 33 & CMS 234A x GP-5 & $1.20^{*}$ & $-3.38^{*}$ & $-7.06 * *$ & $-2.14 * *$ & $-1.08 * *$ & $-0.93 * *$ & $-0.49 * *$ & $0.77 * *$ & $94.12 * *$ & $2.00 * *$ & 0.40 & $46.62 * *$ \\
\hline 34 & CMS 234A x DSR-107 & $-2.43^{* *}$ & $-3.50 *$ & $5.80 * *$ & $-0.75^{* *}$ & 0.26 & $0.72 * *$ & $0.26 * *$ & 0.13 & 16.18 & -0.38 & $-1.43^{* *}$ & $-33.43 * *$ \\
\hline 35 & CMS 234A x NS-15 & $2.08 * *$ & -0.63 & $-1.26 *$ & $-1.22 * *$ & 0.71 & $3.19 * *$ & $1.01^{* *}$ & 0.31 ** & $-37.28 * *$ & $1.32 * *$ & 0.45 & $22.82 *$ \\
\hline 36 & CMS 234A x DSR-35 & 0.33 & -1.25 & $-5.847 * *$ & $3.16^{* *}$ & $1.17 * *$ & $-0.52 * *$ & -0.11 & $-1.56^{* *}$ & $-190.07 * *$ & $-2.22 * *$ & -0.17 & $-73.64 * *$ \\
\hline 37 & CMS 234A x DSR-37 & 0.83 & $-3.88 *$ & -0.12 & $1.95 * *$ & 0.11 & $-0.89 * *$ & $-0.29 * *$ & $-1.54 * *$ & $-188.52 * *$ & 0.69 & $-1.21 * *$ & $-97.49 * *$ \\
\hline 38 & CMS 234A x IB-60 & 0.58 & -1.38 & $11.87 * *$ & $-1.40 * *$ & $-2.76^{* *}$ & $0.76^{* *}$ & 0.03 & $2.61 * *$ & $318.71 * *$ & -0.26 & $2.55^{* * *}$ & $173.44 * *$ \\
\hline 39 & CMS 234A x IB-03 & $-5.93 * *$ & -2.75 & $8.82 * *$ & $1.15 * *$ & 0.54 & $1.71 * *$ & $0.28 * *$ & $2.59 * *$ & $317.18 * *$ & 0.12 & $1.81 * *$ & $164.79 * *$ \\
\hline 40 & CMS 234A x IB-104 & -1.05 & -2.5 & $-5.24 * *$ & $-4.07 * *$ & -0.37 & $1.09 * *$ & $0.63 * *$ & $-2.87 * *$ & $-350.50 * *$ & $-1.49 * *$ & $-1.06^{* *}$ & $-155.26^{* *}$ \\
\hline
\end{tabular}
* - Significant at $5 \%$
** - Significant at $1 \%$ 
Among the testers, IB-104 was the best combiner as it recorded higher gca effect in desired direction for plant height $(\mathrm{cm})$, head diameter $(\mathrm{cm})$, volume weight $(\mathrm{g} / \mathrm{cc})$, test weight $(\mathrm{g})$, seed yield per plant $(\mathrm{g})$, seed yield $(\mathrm{kg} / \mathrm{ha})$ and oil yield $(\mathrm{kg} / \mathrm{ha})$. Hence, it recorded high GCA status. The line IMS 852A and tester NS-15 were observed as good general combiners for oil content (\%). As sunflower is an oilseed crop, there is a need for combining both seed yield and oil content in hybrids. When both seed yield and oil content were considered together, none of the CMS lines have been a good general combiner. Among the testers, only NS-15 found to be the good combiner for both oil content and seed yield.

Considering the gca effect of parents, the female line CMS-234A and male parent IB-104 were found to be the good general combiners for seed yield and its component traits. These results are in accordance with reports of Khan et al., (2008) and Kanwal et al., (2016). The parents, which were good general combiners for economic traits, may be extensively used in hybridization programmes.

The sca effects (Table 3) showed that, none of the hybrids recorded higher sca effects for all the traits. The hybrid IMS 852A $\times$ DSR-37 recorded significant sca effect for all the traits except days to maturity. Considering sca effects, this hybrid found to be good specific combiner. Hence improvement in individual components and certain associated traits may be considered as a desirable approach. The hybrid FMS 852A $\times$ RHA-1-1 recorded higher negative significant sca effect for days to maturity indicating the early maturity of the hybrid compared to parents. The hybrid CMS 302A $\times$ RHA-1-1 recorded higher significant positive sca effects for seed yield per plant $(\mathrm{g})$ and seed yield (kg/ha). Higher significant positive sca effect for oil content recorded by IMS 852A x IB-104. The traits like volume weight $(\mathrm{g} / \mathrm{cc})$, seed yield per plant $(\mathrm{g})$, seed yield (kg/ha), hull content (\%) and oil yield $(\mathrm{kg} / \mathrm{ha})$, the high $\times$ high or low $\mathrm{x}$ low gca combination of parents seemed to be influenced by additive gene action. For the traits like days 50 per cent flowering, days to maturity, plant height $(\mathrm{cm})$, test weight $(\mathrm{g})$ and oil content $(\%)$, the high $\mathrm{x}$ low gca combination of parents was observed to be desirable cross combinations, indicating the predominance of non-additive gene action.

These results are in agreement with the reports of Burli and Jadhav (2001), Sharma et al., (2003), Halaswamy et al., (2004), Reddy and Madhavilatha, (2005), Vishwanath and Goud (2006), Sujatha and Reddy (2009), Keerthi (2010) and Reddy (2012). It is desirable when the crosses showing high sca effect also have parents with high gca effects, in which both additive and non-additive gene actions can be utilized (Kadkol et al., 1984).

In the majority of the crosses high sca effect was due to low $\times$ low, high $\times$ low and low $\times$ high combining parents which further substantiate the operation of non-additive gene action for the characters studied. Therefore it can be concluded in the present investigation that almost all the characters studied were governed by non-additive gene action. Besides using diverse CMS sources are equally efficient in expressing their fullest potential of yield and yield contributing characters. Thus breeders can safely use these sources to broaden the genetic base of CMS source and sunflower crop can be safeguarded from any eventuality of biotic and abiotic threats in future.

\section{Acknowledgement}

The authors are thankful to the Directorate of Oilseeds Research, Hyderabad, India, for providing the germplasm.

\section{References}

Bhargavi, H. A., 2016, Heterosis and combining ability for yield and yield components using newly derived inbred lines of sunflower (Helianthus annuus L.). M. Sc. (Agri) 
Thesis, Univ. Agric. Sci., Dharwad, Karnataka (India).

Burli, A. V. and Jadhav, M. G., 2001, Heterosis and nature of gene effects of oil content and seed filling in sunflower. J. Maharashtra Agric. Univ., 26(3): 326- 327.

Channamma, B. K., 2009, Fertility restoration, Heterosis and Combining ability involving diverse CMS sources in sunflower (Helianthus annuus L.). M. Sc. (Agri.) Thesis, Univ. Agric. Sci., Dharwad (India).

Gilbert, N. E., 1958, Diallel crosses in plant breeding. Heredity, 12: 447-492.

Halaswamy, K. M., Channakrishnaiah, K. M. and Kulkarni, R. S., 2004, Combining ability in sunflower (Helianthus annuus L.). Crop Res., 28(1): 103-112.

Kadkol, G. P., Anand, I. J. and Sharma, R. P., 1984, Combining ability and heterosis in sunflower. Indian J. Genet., 44: 447-451.

Kang, S. A., Khan, F. A., Ashan, M. Z., Chatha, A. S. and Frusat, 2013, Estimation of combining ability for the development of hybrid genotypes in Helianthus annuus L. J. Biol. Agric. Health Care, 3(1): 68-74.

Kanwal, N., Sadaqat, H. A., Ali, Q., Ali, F., Bibi, I. and Niazi, N. K., 2016, Role of combining ability and heterosis in improving achene yield of Helianthus annuus L. An overview. Nature and Science 2016: 14(1).

Keerthi, C. M., 2010, Heterosis and combining ability for yield and yield component traits in sunflower (Helianthus annuus L.). M. Sc. (Agri.) Thesis, Univ. Agric. Sci., Dharwad, Karnataka (India).

Khan, H. U., Rahaman, H., Ahmad, H., Alli, H. and Alam, M., 2008, Magnitude of combining ability of sunflower genotypes in different environments. Pak. J. Bot., 40(1): 151-160.

Khandagale, S. G., Ghodke, M. K. and Khandagale, V. G., 2014, Estimation of combining ability studies in sunflower (Helianthus annuus L.). Asian J. Sci. Technol., 5: 114-116.

Mohanasundaram, K., Manivannan N. and Vindhiya, V. P., 2010, Combining ability analysis for seed yield and its components in Sunflower (Helianthus annuus L.). Electronic Journal of Plant Breeding, 1(4): 864-868.

Reddy P. V. R., 2012, Genetic analysis of seed yield and its component traits and resistance to Alternaria blight in sunflower (Helianthus annuus L.).M. Sc. (Agri.) Thesis, Univ. Agric. Sci. Dharwad, Karnataka (India).

Reddy, A., and Madhavilatha, K., 2005, Combining ability for yield and yield components in sunflower. J. Res., Acharya N. G. Ranga Agric. Univ., 33(2): 12-17.

Sharma, S., Bajaj, R. K., Kaur, N. and Seghal, S. K., 2003, Combining ability studies in sunflower (Helianthus annuus L.). Crop Improv., 30(1): 69-73.

Sprague, G. F. and Tatum, L. A., 1942, General and specific combining ability in single crosses of corn. J. Am. Soc. Agron., 34: 923-932.

Sujatha, M. and Vishnuvardhan Reddy, A., 2008, Identification of fertility restorers/ maintainers in sunflower (Helianthus annuus L.). J. Oilseeds Res., 25(2): 181182.

Sunflower. Pantanagar J. Res., 2: 144-146.

Vishwanath, S. J. and Shanker G. I., 2006, Combining ability and gene action in sunflower (Helianthus annuus L.). $J$. Oilseeds Res., 23(2): 288-291.

Yogesh, L. N., Gangappa, E., Girish, G. and Manjunath, Y. S., 2007, Combining ability studies in sunflower. Indian J. Crop Sci., 2(1): 239-241.

\section{How to cite this article:}

Sukanya Biradar, A.G. Vijaykumar and Naidu, G.K. 2018. Combining Ability Analysis for Seed Yield and Its Component Traits with Diverse CMS Sources in Sunflower (Helianthus annuus L.). Int.J.Curr.Microbiol.App.Sci. 7(04): 954-960. doi: https://doi.org/10.20546/ijcmas.2018.704.101 